\title{
Effect of CT imaging on the accuracy of the finite element modelling in bone
}

\author{
Emir Benca ${ }^{1 *}\left(\mathbb{D}\right.$, Morteza Amini ${ }^{2,3}$ and Dieter H. Pahr ${ }^{2,3}$
}

\begin{abstract}
The finite element (FE) analysis is a highly promising tool to simulate the behaviour of bone. Skeletal FE models in clinical routine rely on the information about the geometry and bone mineral density distribution from quantitative computed tomography (CT) imaging systems. Several parameters in $\mathrm{CT}$ imaging have been reported to affect the accuracy of FE models. FE models of bone are exclusively developed in vitro under scanning conditions deviating from the clinical setting, resulting in variability of FE results $(<10 \%)$. Slice thickness and field of view had little effect on FE predicted bone behaviour $(\leq 4 \%)$, while the reconstruction kernels showed to have a larger effect $(\leq 20 \%)$. Due to large interscanner variations ( $\leq 20 \%$ ), the translation from an experimental model into clinical reality is a critical step. Those variations are assumed to be mostly caused by different "black box" reconstruction kernels and the varying frequency of higher density voxels, representing cortical bone. Considering the low number of studies together with the significant effect of CT imaging on the finite element model outcome leading to high variability in the predicted behaviour, we propose further systematic research and validation studies, ideally preceding multicentre and longitudinal studies.
\end{abstract}

Keywords: Bone and bones, Cortical bone, Finite element analysis, Models (theoretical), Tomography

\section{Key points}

- Several potential sources in computed tomography (CT) affect the accuracy of finite element (FE) bone models.

- Different reconstruction kernels lead to the variability of FE estimated bone behaviour up to $20 \%$.

- Using multiple CT imaging systems might result in large variations, which is especially problematic in multicentre and longitudinal studies.

- Further research is needed before FE models can be incorporated in clinical routine.

\footnotetext{
* Correspondence: emir.benca@muv.ac.at

This is the third article of a thematic series entitled "Advances in MSK imaging and imaging-guided interventions" (Guest Editors Prof. Luca Maria Sconfienza (Milan/IT) and Prof. Franz Kainberger (Vienna/AT).

1 Department of Orthopedics and Trauma Surgery, Medical University of

Vienna, Währinger Gürtel 18-20, 1090 Vienna, Austria

Full list of author information is available at the end of the article
}

\section{Background}

The finite element (FE) analysis is a computer simulation method, originally developed to solve complex problems in civil and aeronautical engineering. Today, this method is widely applied in mathematics and many engineering fields. In this numerical method, a large system is subdivided into a mesh of smaller and simpler parts, the so-called finite elements, through a discretisation process. Force or displacement boundary conditions are then applied at each element's nodes with a material model defined for the analysis domain. This information is used to assemble a large system of equations. Solving the local equations delivers model deformations and internal mechanical stresses.

Applications of the finite element method (FEM) span across a wide range of medical areas overcoming challenges posed by the geometric complexity of biological systems. Geometrical precision is of great importance in orthopaedics and trauma surgery, where FEM is gaining popularity. Another important advantage is the availability of relatively fast and easy image-based model generation approaches. Such models have been used to 
predict bone strength [1-3], evaluate osteosynthesis- [4] and soft-tissue implants [5], investigate the effect of metastatic lesions on the bone's biomechanical behaviour $[6,7]$ and many other subjects. Extensive research has been conducted to estimate the accuracy of FEM predicted bone behaviour. This included effects caused by automated model generation [8], mesh size [9], computed tomography (CT)-derived estimation of material properties [10], micromotion [11], asymmetric tissue behaviour [12], and boundary conditions [9], including different loading scenarios [13], to name few.

The FE bone models are constructed based on medical images. Among imaging modalities, $\mathrm{CT}$ and magnetic resonance imaging provide three-dimensional data while radiography [14] and dual-energy x-ray absorptiometry [15] provide two-dimensional data. Less clinically available highresolution varieties including high-resolution computed tomography and high-resolution magnetic resonance imaging provide enhanced data on structural properties. Micro-CT reveals microstructural details of the human bone but it is principally applicable in vitro only [16]. At present, quantitative computed tomography (QCT) represents the method of choice for the generation of subject-specific finite element models of bone. Finite element method (FEM) in orthopaedics and trauma surgery can be justified if the information provided can improve implantation, monitoring or treatment planning success. Use of ionising radiation for capturing the images required for FEM is associated with a stochastic risk of radiation-induced cancer. Therefore, a robust FE model is required to be optimised to maintain the balance between the lowest radiation exposure, the highest clinical value, and an acceptable accuracy and precision. Among all CT imaging systems, QCT requires minimal tube current time (mAs), keeping the amount of radiation to the patient low. Nevertheless, low-exposure parameters could cause a substantial drop in image quality [17]. FEM can also be time-consuming and costly. Finally, a favourable benefitrisk ratio must be met, especially if alternative methods to the FEM (e.g., dual-energy x-ray absorptiometry in osteoporotic fracture risk prediction) exist [18].

The local material properties of bone are typically defined by converting voxel-specific $\mathrm{CT}$ attenuation values used to characterise tissue, in Hounsfield unit (HU), to bone mineral density (BMD) values $(\rho=\mathrm{a} \times \mathrm{HU}+\mathrm{b})(\rho \ldots$ density, a and $b$... constants) ( $\rho_{\mathrm{QCT}}$ if phantom-calibrated) [19]. Such conversion equations are derived using a calibration phantom placed within the scan field of view (FOV). A voxelspecific Young's modulus (E), which is a material property, is then derived from site-specific empirically determined density to elastic modulus relationships $\left(E=\alpha \times \beta^{\beta}\right.$, with $\alpha$ and $\beta$ being experimentally derived parameters) $[19,20]$.

Such relationships consider bone as a simplified isotropic material since clinical imaging systems do not accurately reveal the microstructural details of the bone necessary to reflect its true anisotropic behaviour. Attempts to extract anisotropic material models from the clinical CT data revealed subtle improvements in the FEM predictions while imposing higher modelling costs $[21,22]$. Furthermore, the mechanical properties of bone, including the elastic modulus, depend on the anatomic site as well. However, experimental procedures to develop E-BMD relationships are sometimes not feasible to be carried out in specific sites (e.g., femoral neck). Inaccuracies in measured HU or calculated BMD will result in an inaccurate derivation of material properties and, consequently, in an inaccurate FE model. Small inaccuracies in the measurement of $\mathrm{HU}$ or calculation of BMD might be neglected in radiological diagnosis in clinical routine. However, in FEM, such deviations from true values are magnified during derivation of material properties through exponential functions. Consequently, large errors are imposed on the simulated biomechanical behaviour of bone [20].

Depending on the structure of interest, different scanning settings might be applied, including the FOV, image resolution and reconstruction kernel. Different scanning settings might as well be applied among patients for the same region of interest, but depending on the patient's size and physique. Deviations from a standard scanning protocol could result in inaccuracies of the FE models. However, these deviations might be inevitable if associated with the specific physician's request in which case the FE model requires a high level of robustness in clinical routine applications. Additional challenges associated with multicentre and longitudinal QCT studies arise from the inherent differences in QCT-derived BMD which also influence related methods like computed tomography $\mathrm{x}$-ray absorptiometry or FEM.

Intrascanner variations (different measurements on the same scanner) and interscanner variations (measurements on different scanners) have a strong impact on CT numbers and derived values. Studies showed that substantial intrascanner and interscanner variabilities are observed for CT attenuation values between different manufacturers' multislice scanners [23].

Therefore, it is crucial to reduce the variations caused by the use of different calibration phantoms, scan settings, image reconstruction and QCT imaging systems but also be aware of the precision of the model given the residual variations (Fig. 1). Table 1 shows the variability of FE results derived from different $\mathrm{CT}$ scanning modalities or the use of different imaging systems. The observed high variability of up to $18 \%$ necessitates for detailed insight into how CT imaging affects the overall model accuracy. This paper aims to discuss in detail the effect of CT imaging, i.e., in situ versus in vitro scanning, calibration differences and intra- and interscanner variability, on the accuracy of $\mathrm{FE}$ modelling in bone. 


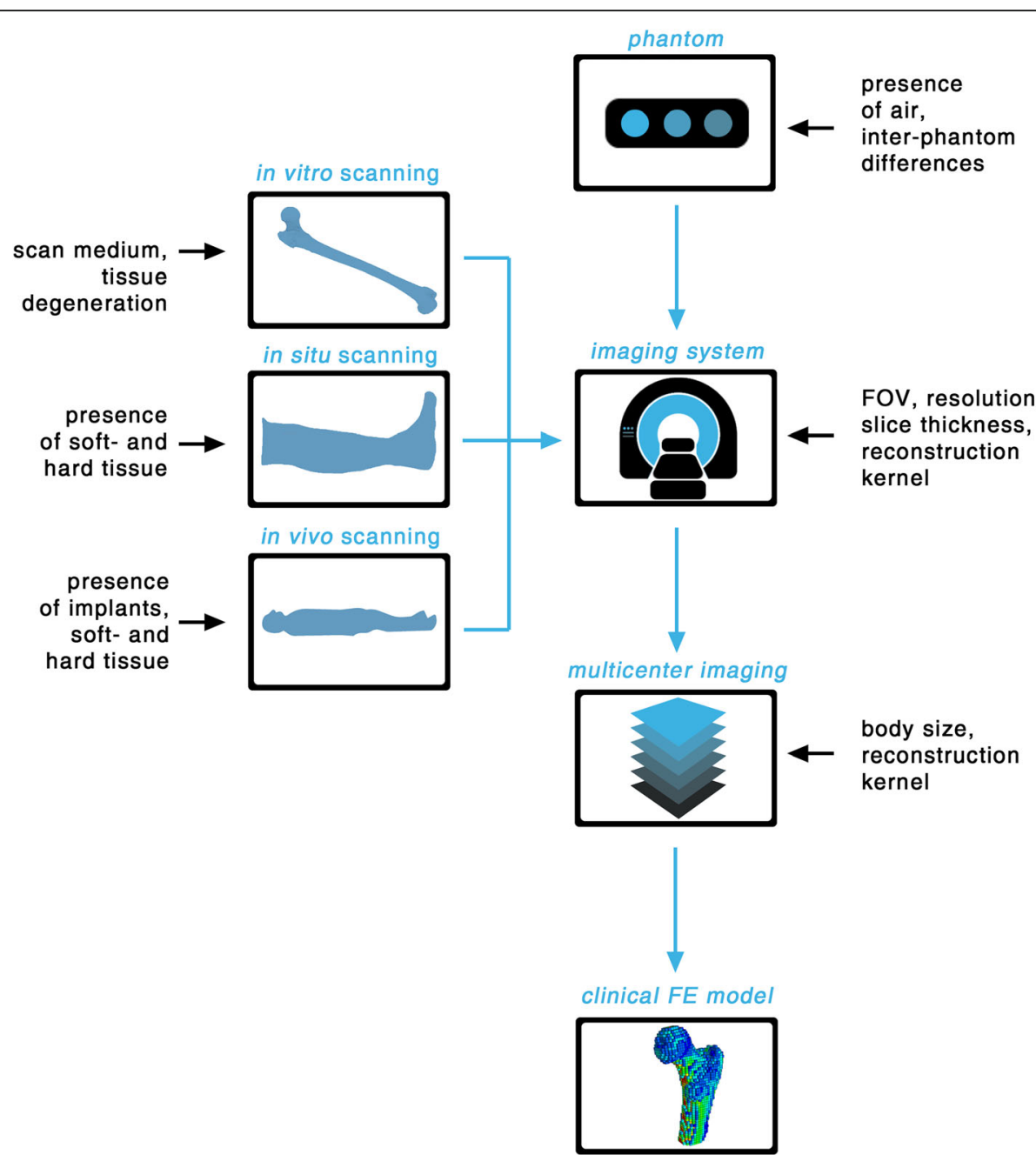

Fig. 1 Overview of potential sources for variations in quantitative computed tomography-based modelling of bone for in vitro, in situ and in vivo scanning reported in the literature. FE, Finite element; FOV, Field of view

\section{In situ versus in vitro scanning}

Performing various medical scans on patients has welldefined protocols. In contrast, scanning anatomic samples, in situ or in vitro, which is the main approach in FEM validation studies, has no defined standard procedure. As a result, the scanning process until extraction of
BMD values based on $\mathrm{HU}$ can be affected by multiple parameters. Any deviation from the clinical environment could potentially be a source of variation in the estimated behaviour of the sample. Sample preparation parameters like presence and amount of soft tissue, presence of other limbs or anatomical structures within

Table 1 Reported variability of finite element results as an effect of different computed tomography parameters

\begin{tabular}{|c|c|c|c|c|c|}
\hline Authors, year [reference] & Comparison between & Specimens & Number & Variables & Variability \\
\hline Keyak and Falkinstein (2003) [23] & In situ versus in vitro (water) & Femur & 2 & Ultimate load & $5.2 \%$ and $13.3 \%$ \\
\hline Carpenter et al. (2014) [7] & $\mathrm{CT}$ scanners & Femur & 20 & Ultimate load & $12.5 \%(\mathrm{CV})$ \\
\hline Eggermont et al. (2018) [14] & CT scanners & Femur & 6 & Ultimate load & Maximum 17\% \\
\hline Eggermont et al. (2018) [14] & Slice thickenss & Femur & 6 & Ultimate load & Maximum 4\% \\
\hline Eggermont et al. (2018) [14] & Field of view & Femur & 6 & Ultimate load & Maximum 4\% \\
\hline Eggermont et al. (2018) [14] & Reconstruction kernels & Femur & 6 & Ultimate load & Maximum 9\% \\
\hline Michalski et al. (2019) [30] & Reconstruction kernels & Femur & 1 & Ultimate load & $18.2 \%$ \\
\hline Michalski et al. (2019) [30] & Reconstruction kernels & Femur & 1 & Stiffness & $16.5 \%$ \\
\hline
\end{tabular}


the FOV, presence and location of a phantom and scanner settings are some major examples.

The vast majority of published FE models of bone are based on human specimens scanned in vitro with soft tissue removed for easier handling or instrumentation $[2,4,6]$. CT scanning of isolated bone specimens does not only facilitate the image segmentation but also eliminates additional sources of error that are due to increased noise, streak artefacts, and beam hardening caused by the presence of other skeletal elements, implants or large amounts of soft tissue within the FOV. This is typically problematic in obese patients or when scanning the region of interest is shielded by other large skeletal elements such as the humeral or femoral head.

Sitzer et al. [24] reported differences in femoral diaphysis BMD measurements via QCT imaging $(n=3)$ of up to $17.5 \%$ when scanned in air and water compared to in vivo. Similarly, Keyak and Falkinstein [25] compared the FE predicted fracture loads of human femora scanned in situ and in vitro. Following in situ scanning, the femora $(n=2)$ were then retrieved, stripped of soft tissue and scanned immersed in water to reduce artefacts using the same scanning settings. The crosssectional areas and cross-sectional densities varied between the two scanning conditions $(<14 \%$ and $22 \%$, respectively), in opposite directions. The differences in areas and densities for the two subjects were also opposite in sign. Lower failure loads $(5.2 \%$ and $13.3 \%$ difference) were also reported in models based on in vitro scans compared to data obtained in situ [25]. In another study, lower failure loads $(8 \pm 3 \%)$, along with lower HU $(<3 \%)$ and BMD $(<7 \%)$, have been reported in case of presence of air between the patient (anatomic specimens) and the calibration phantom $(n=6)$ [26].

Furthermore, decomposition of biological tissue causes the generation of gasses in vitro, which are then stored in the bone marrow and intratrabecular spaces in specimens, causing imaging artefacts [25]. Therefore, it is recommended to scan anatomic specimens submerged in water and followed by evacuation of the residual air to minimise the artefacts. Authors should be aware of the ideal scanning conditions given in vitro experimental setting, which might strongly differ from clinical settings (e.g., presence of metal implants, obese patients, and children) that distort the model's precision. In a preliminary parametric study on a single femoral specimen, it was shown how selected scan parameters might affect the calculated BMD of the sample [27]. A range of common parameters involved in the extraction of BMD values from the measured $\mathrm{HU}$ in $\mathrm{CT}$ scans were studied (including FOV, tube current, reconstruction filter, source filter, scan repetition, phantom/bone presence, and target bone positioning). Presence of high-density elements (another limb/bone, high-density phantom, implant, etc.) was reported to have the highest potential to distort the BMD calculations, followed by vertical misplacement of the bone and reconstruction filter [27].

\section{Calibration variability}

As mentioned earlier, the mechanical properties of the QCT-based FE models are based on the calculated BMD values extracted from QCT images. The linear attenuation coefficient measurement is transformed into the linear Hounsfield unit scale. Usually, the HU values measured by CT scanners are calibrated with respect to water, defined as $0 \mathrm{HU}$. Since the linear $\mathrm{x}$-ray attenuation depends on many factors (electron density, atomic composition of the matter, photon energy spectrum, geometrical configuration of the phantom, detector sensitivity, and reconstruction algorithm) [28], such HU values need to be further calibrated for each biological tissue of interest. Typically, bone may range from 100 up to $1,400 \mathrm{HU}$ [29]. Dense cortical bone can reach values above $1,800 \mathrm{HU}$ [29].

Different calibration procedures can be applied [30]: in-scan calibration where simultaneously a phantom is scanned with the subject, asynchronous calibration where the patient and phantom scans are done separately and phantom-less or internal calibration where internal tissue is used for the calibration (e.g., air, fat, and muscle) [31]. All techniques have various shortcomings. For example, in the case of in-scan calibration, a phantom is used during the scan, which possibly influences the back projection. Asynchronous calibration needs stable scanners and periodic recalibration. If scanner stability is maintained, asynchronous calibration is a convenient way.

A recent study showed that asynchronously calibrated QCT measurements provide results comparable to the established synchronously (in-scan) calibrated QCT measurements $\left( \pm 1.5 \mathrm{mg} / \mathrm{cm}^{3}\right.$ for vBMD) [32]. In both methods, phantoms are used which are based on CT measurements of liquid or solid bone equivalent materials with known BMD values. Examples are calcium hydroxyapatite $\left(\mathrm{CaCO}_{3}\right.$ or $\left.\mathrm{CaHA}\right)$ or hydrogen dipotassium phosphate $\left(\mathrm{K}_{2} \mathrm{HPO}_{4}\right.$ or KHP) [31]. Sande et al. [33] scanned four different phantoms (Catphan phantom (The Phantom Laboratory, Salem, NY, USA)) on one QCT scanner to examine interphantom variations. Only minor differences in measured HU (i.e., 2-5 HU) were identified.

Since in routine clinical CT scans, no phantoms are typically present, and phantom-less calibration methods have been investigated in the literature. This could be achieved by registration of air and/or specific tissues, such as fat or muscle, with known densities, to their equivalent HU. A study indicates that phantomequivalent measurements can be reliably obtained from 
CT scans using patient-specific phantom-less calibration without any significant effect on simulated bone's strength $\left( \pm 10 \mathrm{~N}\right.$ for strength, $\pm 1 \mathrm{mg} / \mathrm{cm}^{3}$ for vBMD (volumetric bone mineral density); $\pm 0.001 \mathrm{~g} / \mathrm{cm}^{2}$ for aBMD (areal bone mineral density)) [34]. These findings are encouraging in terms of the feasibility to process valuable existing clinical QCT data acquired without a calibration phantom. This is of importance in retrospective or longitudinal studies.

\section{Intrascanner variability}

In terms of intrascanner variability, deviating from a standard clinical protocol in CT settings is another potential source of variability in the representation of bone morphology. This might consequently put the accuracy of the finite element model in jeopardy. Examples of such parameters are the FOV, reconstruction kernel, tube current and resolution. Eggermont et al. [26] showed that slice thickness and field of view (FOV) had little effect on FE predicted failure loads $(\leq 4 \%)$ in metastatic femora $(n=6)$, while the reconstruction kernels showed to have a larger effect on the failure loads $(\leq 8 \%)$.

Michalski et al. [20] performed eighteen in vivo scans of a proximal femur. They found even higher mean differences in both, the simulated stiffness and ultimate load when using a bone reconstruction kernel, compared to the standard kernel. The mean absolute percent difference was $16.5 \%$ for stiffness and $18.2 \%$ for failure load. At the same time, the overall volumetric bone mineral density (vBMD) and bone mineral content were increased, but at a lower magnitude $(\leq 7 \%)$. The histogram analysis revealed that the bone kernel increased the frequency of higher density voxels, representing cortical bone. A thicker cortex will increase the bone mineral content and consequently the density, which will have a stronger positive effect on bone's strength due to the cortex' dominance in load-bearing (over 90\% in femora). The use of a calibration phantom was not able to correct the effect of changes in reconstruction kernel in most CT scanners $[20,26,35]$.

The preliminary results of Amini et al. [27] also indicated negligible effects of FOV, tube current and scan filters on the BMD (\% absolute difference between paired scans of $<2 \%$ ). Application of bone reconstruction kernel had 3.3\% effect on the BMD. An intrascanner variability in failure load and stiffness was observed in FE models based on low resolution and high-resolution QCT data [36]. This is especially important since an FE model based on high-resolution QCT parameters, as often performed in initial validation studies, may not translate well to models obtained at lower resolution using same FE parameters when processing clinical scans.

\section{Interscanner variability}

Once developed and validated, an FE model would ideally be applied in multicentre and longitudinal studies where two or more QCT imaging systems would be used to assess patient-specific mechanical behaviour of specific skeletal structures. One of the challenges confronting the examiners is how to account for the inherent differences in BMD and bone strength parameters that may exist between the QCT imaging systems [37]. Thus, the ability to pool data or compare measurements conducted at different time points would be affected.

A recent study compared the failure loads in metastatic femora based on calibrated QCT scans obtained in four different radiotherapy institutes in the Netherlands and three different QCT imaging systems at constant settings [26]. The differences between imaging systems varied significantly: up to $7 \%$ in the cortical $\mathrm{HU}, 6 \%$ in the trabecular $\mathrm{HU}, 6 \%$ in the cortical BMD and $12 \%$ in the trabecular BMD. The variations in bone morphology resulted in up to $17 \%$ variation in ultimate load. Another study assessed scan data of a phantom on eight different $\mathrm{CT}$ imaging systems and found the minimum variation to be $7 \mathrm{HU}$ and the largest $56 \mathrm{HU}$ [33]. The variations were the largest for materials at extremities of the $\mathrm{HU}$ spectrum. In other studies, the largest interscanner deviations have also been observed in higher $\mathrm{HU}$ values [33, 38-40]. Additionally, one study reported the effect of body size on interscanner differences. An increased body size tends to decrease the cortical BMD reducing the bone's load-bearing ability [37]. This is likely a result of increased beam hardening as more material is present in the scanner FOV.

\section{Further considerations}

QCT-based finite element models can accurately predict deformations of human bone with a root mean squared error (normalised by the peak measured strain) of only $7 \%$ [10] and its strength of 15-16\% standard error of the estimate, normalised by the average measured strength [18]. The use of an FE model in clinical routine is primarily justified if the clinical advantage is relevant taking into account the level of radiation exposure. It is important to stress that the gain in accuracy has to be of clinical significance rather than a result of statistical analysis. Therefore, the inaccuracies resulting from CT imaging must not compromise the overall accuracy and robustness of FE model to the extent that its application has no added value to the diagnosis or treatment. Many skeletal FE models rely on laws linking the material properties, such as the Young's modulus to bone mineral density. Therefore, the precise measurement of the patient's BMD will have a direct effect on the model's accuracy and its prediction power $[10,18]$. 
The accuracy of a QCT-based FE model is affected by the amount of noise, streak artefacts and beam hardening during the imaging process. With the increase of these sources of image degradation, the accuracy of the CT scan-derived material properties and geometry, and consequently, the accuracy of the skeletal FE models decrease. This is especially challenging in clinical routine. The literature on the effect of CT imaging on the finite element accuracy in skeletal modelling is scarce, and conclusions are often based on small study populations and low statistical power $[1,24]$. Most studies investigate the effect of different scan parameters and their effect on HU and BMD. Only a few studies have investigated the direct effect of single parameters on the variability of FE results (Table 1). Nevertheless, several studies report statistically-and, more importantly, clinically significant differences in FE results when using different scan protocols, scan resolution and different QCT imaging systems, ranging from 4 to $18 \%$ variability. This indicates that, in the clinical setting, different clinics could potentially obtain distinctly dissimilar predictions of bone strength and stiffness if using different QCT imaging systems and/or if handling the QCT settings and image reconstructions differently. Among all investigated parameters, the use of different CT scanners causes the highest variations in FE-derived estimation of bone's biomechanical behaviour [20, 26, 31, 37]. Previous assumptions that differences between QCT imaging systems and protocols or other varying parameters could be corrected when using a calibration phantom $[41,42]$ were refuted with more recent studies [26, 33, 35, 37]. FOV and slice thickness have a minor, but significant effect on the HU and FE predictions [26, 39].

The main source of inaccuracy seems to be the reconstruction kernel, which has a strong effect on the HU [38, 39]. This effect is the largest for the lower and higher values in the $\mathrm{HU}$ spectrum. The precise cause of this effect remains unknown and unexplored since reconstruction kernels are manufacturer specific black boxes. The use of different reconstruction kernels is critical, especially in strength prediction, since they mainly affect the frequency of higher density voxels, which represent the load-bearing cortex of the bone.

The translation from an experimental model into clinical reality is a critical step, which requires carefully conducted validation studies. Several studies have shown high variations in BMD for in situ and in vitro scans. In vitro measurements are prone to errors arising from the partial volume effect, with underestimation of BMD resulting from the inclusion of air as well as bone in a single voxel [43]. This is especially problematic in regions dominated by trabecular bone. Thus, when scanned in vitro, specimens should be submerged in water. It should be still noted that available research on the effect of different scanning conditions on the accuracy of FE models is currently rather explorative rather than systematic and based on data obtained on a very low number of specimens $(n=2-6)$ [24-26].

Furthermore, the presence of air gaps must be avoided as air gaps between the patient and phantom showed to cause different scanner responses [39]. Ideally, calibration should be performed using slices without an air gap in them. This is important when cushions are used to provide comfort for the patient during scanning. Resulting air gaps lead to shading artefacts and affect the calibration curve [39]. The presence of hard tissue, especially the pelvis, and soft tissue and increased body size are sources of more noise, streak artefacts and beam hardening. They, in turn, result in a decrease of both, the CT-derived material properties and geometry. Consequently, the accuracy and reliability of the corresponding FE models decrease. Therefore, it is crucial to take the non-ideal in vivo scanning conditions into account and apply them in vitro where possible. FE models constructed based on optimised high-resolution QCT parameters may not necessarily be useful since typically available clinical scans are assessed at a lower resolution.

\section{Conclusion}

We showed that while QCT-based FE models have a great potential for use in clinical routine, their in vivo results must be interpreted carefully, with special attention given to subject size, resolution and quality of CT-scan images, and especially when using multiple QCT imaging systems. Further, improvements are necessary to increase the robustness in their predictive power on different QCT imaging systems and under different settings. Development of standards to handle in situ and in vitro scans, as well as the availability of all relevant details of the scanning and model generation details in scientific reports, are necessary to facilitate the transition of FE-based predictions from research to clinics.

\section{Abbreviations \\ aBMD: areal bone mineral density; BMD: Bone mineral density; CT: Computed tomography; FE: Finite element; FEM: Finite element method; FOV: Field of view; HU: Hounsfield unit; QCT: Quantitative computed tomography; $\checkmark B M D$ : Volumetric bone mineral density}

\section{Authors' contributions}

EB designed the study, performed literature research and drafted and revised the manuscript. MA participated in study design and manuscript drafting and critically revised the manuscript. DHP critically revised the manuscript. All authors read and approved the final manuscript.

\section{Funding information}

The authors state that this work has not received any funding.

\section{Availability of data and materials}

The datasets used and/or analysed during the current study are available from the corresponding author on reasonable request. 


\section{Ethics approval and consent to participate}

Not applicable

\section{Consent for publication}

Not applicable.

\section{Competing interests}

The authors declare that they have no competing interests.

\section{Author details}

'Department of Orthopedics and Trauma Surgery, Medical University of Vienna, Währinger Gürtel 18-20, 1090 Vienna, Austria. ${ }^{2}$ Institute of Lightweight Design and Structural Biomechanics, TU Wien, Getreidemarkt 9 1060 Vienna, Austria. ${ }^{3}$ Division Biomechanics, Karl Landsteiner University of Health Sciences, Dr.-Karl-Dorrek-Straße 30, 3500 Krems an der Donau, Austria.

Received: 27 March 2020 Accepted: 15 July 2020

Published online: 01 September 2020

\section{References}

1. Keyak JH, Rossi SA, Jones KA, Skinner HB (1997) Prediction of femoral fracture load using automated finite element modeling. J Biomech 31:125133. https://doi.org/10.1016/S0021-9290(97)00123-1

2. Dall'Ara E, Pahr D, Varga P, Kainberger F, Zysset P (2012) QCT-based finite element models predict human vertebral strength in vitro significantly better than simulated DEXA. Osteoporos Int 23:563-572. https://doi.org/10 1007/s00198-011-1568-3

3. Zysset PK, Dall'Ara E, Varga P, Pahr DH (2013) Finite element analysis for prediction of bone strength. BoneKEy reports 2. doi: 10.1038\%2Fbonekey.2 013.120

4. Baumbach SF, Synek A, Traxler H, Mutschler W, Pahr D, Chevalier Y (2015) The influence of distal screw length on the primary stability of volar plate osteosynthesis - a biomechanical study. J Orthop Surg Res 10:139. https:// doi.org/10.1186/s13018-015-0283-8

5. Shriram D, Kumar GP, Cui F, Lee YHD, Subburaj K (2017) Evaluating the effects of material properties of artificial meniscal implant in the human knee joint using finite element analysis. Sci Rep 7:1-11. https://doi.org/10. 1038/s41598-017-06271-3

6. Benca E, Synek A, Amini M et al (2019) QCT-based finite element prediction of pathologic fractures in proximal femora with metastatic lesions. Sci Rep 9:1-9. https://doi.org/10.1038/s41598-019-46739-y

7. Eggermont F, van der Wal G, Westhoff Pet al (2020) Patient-specific finite element computer models improve fracture risk assessments in cancer patients with femoral bone metastases compared to clinical guidelines. Bone 130:115101. doi:https://doi.org/10.1016/j.bone.2019.115101

8. Taddei F, Cristofolini L, Martelli S, Gill HS, Viceconti M (2006) Subject-specific finite element models of long bones: An in vitro evaluation of the overall accuracy. J Biomech 39:2457-2467. https://doi.org/10.1016/j.jbiomech.2005 07.018

9. Jacobs CR, Davis BR, Rieger CJ, Francis JJ, Saad M, Fyhrie DP (1999) The impact of boundary conditions and mesh size on the accuracy of cancellous bone tissue modulus determination using large-scale finiteelement modeling. J Biomech 32:1159-1164. https://doi.org/10.1016/S00219290(99)00115-3

10. Schileo E, Dall'Ara E, Taddei F et al (2008) An accurate estimation of bone density improves the accuracy of subject-specific finite element models. J Biomech 41:2483-2491. https://doi.org/10.1016/j.jbiomech.2008.05.017

11. Viceconti M, Muccini R, Bernakiewicz M, Baleani M, Cristofolini L (2000) Large-sliding contact elements accurately predict levels of bone-implant micromotion relevant to osseointegration. J Biomech 33:1611-1618. https:// doi.org/10.1016/S0021-9290(00)00140-8

12. Niebur GL, Feldstein MJ, Yuen JC, Chen TJ, Keaveny TM (2000) Highresolution finite element models with tissue strength asymmetry accurately predict failure of trabecular bone. J Biomech 33:1575-1583. https://doi.org/ 10.1016/S0021-9290(00)00149-4

13. Grassi L, Schileo E, Taddei F et al (2012) Accuracy of finite element predictions in sideways load configurations for the proximal human femur. J Biomech 45:394-399. https://doi.org/10.1016/j.jbiomech.2011.10.019

14. Langton C, Pisharody S, Keyak J (2009) Comparison of 3D finite element analysis derived stiffness and BMD to determine the failure load of the excised proximal femur. Med Eng Phys 31:668-672. https://doi.org/10.1016/j. medengphy.2008.12.007

15. Naylor KE, McCloskey EV, Eastell R, Yang L (2013) Use of DXA-based finite element analysis of the proximal femur in a longitudinal study of hip fracture. J Bone Miner Res 28:1014-1021. https://doi.org/10.1002/jbmr.1856

16. Feldkamp LA, Goldstein SA, Parfitt MA, Jesion G, Kleerekoper M (1989) The direct examination of three-dimensional bone architecture in vitro by computed tomography. J Bone Miner Res 4:3-11. https://doi.org/10.1002/ jbmr.5650040103

17. Kalender WA, Deak P, Kellermeier M, van Straten M, Vollmar SV (2009) Application- and patient size-dependent optimization of $\mathrm{x}$-ray spectra for CT. Med Phys 36:993-1007. https://doi.org/10.1118/1.3075901

18. Viceconti M, Qasim M, Bhattacharya P, Li X (2018) Are CT-based finite element model predictions of femoral bone strengthening clinically useful? Curr Osteoporos Rep 16:216-223. https://doi.org/10.1007/s11914-018-0438-8

19. Knowles NK, Reeves JM, Ferreira LM (2016) Quantitative computed tomography (QCT) derived bone mineral density (BMD) in finite element studies: a review of the literature. J Exp Orthop 3:36-36. https://doi.org/10. 1186/s40634-016-0072-2

20. Michalski AS, Edwards WB, Boyd SK (2019) The Influence of Reconstruction Kernel on Bone Mineral and Strength Estimates Using Quantitative Computed Tomography and Finite Element Analysis. J Clin Densitom 22: 219-228. https://doi.org/10.1016/j.jocd.2017.09.001

21. Nazemi SM, Cooper DML, Johnston JD (2016) Quantifying trabecular bone material anisotropy and orientation using low resolution clinical CT images: a feasibility study. Med Eng Phys 38:978-987. https://doi.org/10.1016/j. medengphy.2016.06.011

22. Nazemi SM, Kalajahi SMH, Cooper DML et al (2017) Accounting for spatial variation of trabecular anisotropy with subject-specific finite element modeling moderately improves predictions of local subchondral bone stiffness at the proximal tibia. J Biomech 59:101-108. https://doi.org/10. 1016/j.jbiomech.2017.05.018

23. Birnbaum BA, Hindman N, Lee J, Babb JS (2007) Multi--detector row CT attenuation measurements: assessment of intra-and interscanner variability with an anthropomorphic body CT phantom 1. Radiology 242:109-119

24. Sitzer A, Aulmann L, Wendlandt R et al (2015) Evaluation of local alterations in femoral bone mineral density measured via quantitative $\mathrm{CT}$. Current Directions in Biomedical Engineering 1:327-330. https://doi.org/10.1515/ cdbme-2015-0081

25. Keyak JH, Falkinstein Y (2003) Comparison of in situ and in vitro CT scanbased finite element model predictions of proximal femoral fracture load. Med Eng Phys 25:781-787. https://doi.org/10.1016/S1350-4533(03)00081-X

26. Eggermont F, Derikx LC, Free J et al (2018) Effect of different CT scanners and settings on femoral failure loads calculated by finite element models. J Orthop Res 36:2288-2295. https://doi.org/10.1002/jor.23890

27. Amini M, Reisinger A, Pahr D (2019) Effect of selected scan parameters on QCT-based BMD estimations of a femur. Paper presented at the 25th Congress of the European Society of Biomechanics, Vienna, Austria,

28. Celenk C, Celenk P (2012) Computed tomography - clinical applications. InTech

29. Norton MR, Gamble C (2001) Bone classification: an objective scale of bone density using the computerized tomography scan. Clin Oral Implants Res 12 (1):79-84. https://doi.org/10.1034/j.1600-0501.2001.012001079.x

30. Engelke K, Lang T, Khosla S et al (2015) Clinical use of quantitative computed tomography-based advanced techniques in the management of osteoporosis in adults: the 2015 ISCD official positions-part III. J Clin Densitom 18:393-407

31. Eggermont F, Verdonschot $N$, van der Linden $Y$, Tanck E (2019) Calibration with or without phantom for fracture risk prediction in cancer patients with femoral bone metastases using CT-based finite element models. PloS One 14:e0220564. https://doi.org/10.1371/journal.pone.0220564

32. Brown JK, Timm W, Bodeen G et al (2016) Asynchronously calibrated quantitative bone densitometry. J Clin Densitom 20:216-225 https://doi.org/ 10.1016/j.jocd.2015.11.001

33. Sande EPS, Martinsen ACT, Hole EO, Olerud HM (2010) Interphantom and interscanner variations for Hounsfield units-establishment of reference values for HU in a commercial QA phantom. Phys Med Biol 55:5123. https:// doi.org/10.1088/0031-9155/55/17/015

34. Lee DC, Hoffmann PF, Kopperdahl DL, Keaveny TM (2017) Phantomless calibration of $C T$ scans for measurement of BMD and bone strength-interoperator reanalysis precision. Bone 103:325-333 
35. Giambini H, Dragomir-Daescu D, Huddleston PM, Camp JJ, An K-N, Nassr A (2015) The effect of quantitative computed tomography acquisition protocols on bone mineral density estimation. J Biomech Eng 137. https:// doi.org/10.1115/1.4031572

36. Dragomir-Daescu D, Salas C, Uthamaraj S, Rossman T (2015) Quantitative computed tomography-based finite element analysis predictions of femoral strength and stiffness depend on computed tomography settings. J Biomech 48:153-161. https://doi.org/10.1016/j.jbiomech.2014.09.016

37. Carpenter RD, Saeed I, Bonaretti S et al (2014) Inter-scanner differences in in vivo QCT measurements of the density and strength of the proximal femur remain after correction with anthropomorphic standardization phantoms. Med Eng Phys 36 (10):1225-1232. https://doi.org/10.1016/j. medengphy.2014.06.010

38. Birnbaum BA, Hindman N, Lee J, Babb JS (2007) Multi-detector row CT attenuation measurements: assessment of intra- and interscanner variability with an anthropomorphic body CT phantom. Radiology 242:109-119. https://doi.org/10.1148/radiol.2421052066

39. Free J, Eggermont F, Derikx L et al (2018) The effect of different CT scanners, scan parameters and scanning setup on Hounsfield units and calibrated bone density: a phantom study. Biomed Phys Eng Express 4 (5): 055013. https://doi.org/10.1088/2057-1976/aad66a

40. Mackin D, Fave X, Zhang L et al (2015) Measuring CT scanner variability of radiomics features. Invest Radiol 50 (11):757. 10.1097\%2FRLI.0000000000000180

41. Keyak JH, Kaneko TS, Tehranzadeh J, Skinner HB (2005) Predicting proximal femoral strength using structural engineering models. Clin Orthop Relat Res 437:219-228. https://doi.org/10.1097/01.blo.0000164400.37905.22

42. Katz Y, Dahan G, Sosna J, Shelef I, Cherniavsky E, Yosibash Z (2019) Scanner influence on the mechanical response of QCT-based finite element analysis of long bones. J Biomech 86:149-159. https://doi.org/10.1016/j.jbiomech. 2019.01.049

43. Cuppone M, Seedhom BB, Berry E, Ostell AE (2004) The longitudinal Young's modulus of cortical bone in the midshaft of human femur and its correlation with CT scanning data. Calcif Tissue Int 74:302-309. https://doi. org/10.1007/s00223-002-2123-1

\section{Publisher's Note}

Springer Nature remains neutral with regard to jurisdictional claims in published maps and institutional affiliations.

\section{Submit your manuscript to a SpringerOpen ${ }^{\circ}$ journal and benefit from:}

- Convenient online submission

- Rigorous peer review

- Open access: articles freely available online

High visibility within the field

- Retaining the copyright to your article

Submit your next manuscript at $\boldsymbol{\nabla}$ springeropen.com 